\title{
Vacuum stability and the Cholesky decomposition
}

\author{
James Unwin ${ }^{\mathrm{a}}$ \\ Mathematical Institute, University of Oxford, 24-29 St. Giles', Oxford, OX1 3LB, UK
}

Received: 20 February 2011 / Published online: 27 May 2011

(C) The Author(s) 2011. This article is published with open access at Springerlink.com

\begin{abstract}
We discuss how the Cholesky decomposition may be used to ascertain whether a critical point of the field theory scalar potential provides a stable vacuum configuration. We then use this method to derive the stability conditions in a specific example.
\end{abstract}

\section{Introduction}

Understanding the structure of the vacuum is of central importance in quantum field theory. Local minima of the effective quantum potential correspond to stable vacuum configurations of the system and physical states in the quantum theory are identified with fluctuations around a given minimum.

In this note we shall introduce the Cholesky decomposition and present a method for using the Cholesky decomposition of the Hessian of the potential to ascertain the stability of a given vacuum configuration. Whilst approaches of this manner are familiar in some mathematical disciplines, it does not seem to be widely known in theoretical physics.

This technique should be of value in the study of vacuum stability in models of interest in particle physics. In particular, the study of the vacuum structure of gauge mediated supersymmetry breaking models, where stability analysis is often complicated due to the number of scalar fields entering in the potential [1-5].

Firstly, we shall present a summary of the relevant notions from linear algebra and discuss their use in demonstrating vacuum stability. To illustrate this approach, in the latter half of the paper, we shall apply these techniques to a particular example for which vacuum stability has yet to be shown. Additional areas for potential application of this approach are suggested in the concluding remarks.

a e-mail: unwin@maths.ox.ac.uk

\section{The Cholesky decomposition}

Given a general scalar potential $V=V\left(\phi_{1}, \ldots, \phi_{n}\right)$, a stable (degenerate) vacuum configuration of the system is a (semi)stable critical point of $V$. That is a set of parameters for which

$\frac{\partial V}{\partial \phi_{i}}=0 \quad$ for $i=1, \ldots, n$.

The Hessian $H$ of a potential $V\left(\phi_{1}, \ldots, \phi_{n}\right)$ is defined to be the matrix of second derivatives of the potential with respect to each of the fields it depends upon:

$H=\left(\begin{array}{ccc}\frac{\partial^{2} V}{\partial \phi_{1} \partial \phi_{1}} & \cdots & \frac{\partial^{2} V}{\partial \phi_{1} \partial \phi_{n}} \\ \vdots & \ddots & \vdots \\ \frac{\partial^{2} V}{\partial \phi_{n} \partial \phi_{1}} & \cdots & \frac{\partial^{2} V}{\partial \phi_{n} \partial \phi_{n}}\end{array}\right)$.

The Hessian is a useful object since the stability of a given critical point can be inferred from the properties of the matrix. Suppose that the point $p$ is a critical point of $V$, then it is (semi)stable if and only if the Hessian of $V$ at $p$ is positive-(semi)definite [6]. If the Hessian $H$ is singular at $p$, then using row operations $H$ may be separated into a nonsingular symmetric submatrix $M$ and a set of zero vectors. If the submatrix $M$ is positive-definite, then $H$ is positivesemidefinite.

For a Hermitian or real symmetric matrix $A$ the following three statements are equivalent [7]:

(i) $A$ is positive-definite, i.e.

$$
\langle\boldsymbol{x}, A \boldsymbol{x}\rangle>0
$$

for all nonzero vectors $\boldsymbol{x}$.

(ii) All of the eigenvalues of $A$ are positive.

(iii) $A$ can be uniquely decomposed as follows

$A=C^{\dagger} C$

where $C$ is an upper triangular matrix with real strictly positive diagonal entries. 
The factorisation of $A$ into $C$ and its conjugate in (iii) is referred to as the Cholesky decomposition of $A$ and may be considered as the matrix analogue of the square root operation for scalar numbers.

Additionally, the three statements below are also equivalent to each other [7]:

(a) $A$ is positive-semidefinite, i.e.

$$
\langle\boldsymbol{x}, A \boldsymbol{x}\rangle \geq 0
$$

for all nonzero vectors $\boldsymbol{x}$.

(b) All of the eigenvalues of $A$ are non-negative.

(c) There exists a (possibly singular) matrix $B$ such that

$$
A=B^{\dagger} B \text {. }
$$

Whilst one can ascertain whether a matrix is positive(semi)definite by examining its eigenvalues, finding analytically the eigenvalues of $H$ evaluated at $p$ becomes increasingly more difficult as the dimension of the parameter space grows. Demonstrating the existence of the Cholesky decomposition provides an alternative method to finding the eigenvalues of the Hessian and can often be more practical to compute.

Since the Hessian is symmetric, if it is nonsingular at a given critical point $p$ then it can be factorised as $H=U^{\dagger} U$, where $U$ is an upper triangular matrix with symbolic entries. The requirement that the diagonal entries of $U$ be real and positive provides the stability conditions for the vacuum configuration. Note that when the diagonal entries are real and positive, the matrix $U$ is the Cholesky decomposition of $H$. If the Hessian is singular at $p$, then one must inspect the nonsingular submatrix $M$. Similarly, one may factorise $M=U^{\dagger} U$ and the vacuum is semistable if the diagonal entries of $U$ are real and positive.

Note that the dimension of the singular subspace gives the number of massless states, some of which may be Nambu-Goldstone bosons, but others correspond to moduli ('flat-direction' fields in the supersymmetric case).

For a given Hermitian or real symmetric matrix $A$ with symbolic entries, there are algorithms [8], based on iterative elementary row operations, through which one may obtain the factorisation $A=U^{\dagger} U$. In the case where $A$ is positivedefinite this provides the Cholesky decomposition. Moreover, most standard mathematical computing packages can perform this operation via preprogrammed functions $[9,10]$.

\section{A particular example}

We shall illustrate below the method outlined in Sect. 2 by deriving the stability conditions for the vacuum configuration of a model proposed by Chan and Tsou [11], related to the Standard Model. The problem requires the analysis of a
$10 \times 10$ Hessian and thus provides a sufficiently complicated example to demonstrate the utility of our method.

The scalar content of the theory under consideration is as follows: one complex scalar field $\varphi$ transforming in the fundamental representation of su(2) and three complex scalar fields $\phi^{i}(i=1,2,3)$ transforming in the fundamental representation of $\operatorname{su}(3)$. The scalar potential of the model has the following form:

$$
\begin{aligned}
V= & -\mu_{w}|\varphi|^{2}+\lambda_{w}|\varphi|^{4}-\mu_{s} \sum_{a=1}^{3} \sum_{i=1}^{3}\left|\phi_{a}^{i}\right|^{2} \\
& +\lambda_{s}\left(\sum_{a=1}^{3} \sum_{i=1}^{3}\left|\phi_{a}^{i}\right|^{2}\right)^{2}+\kappa_{s} \sum_{a, b=1}^{3}\left|\phi_{a}^{i} \cdot \phi_{b}^{i *}\right|^{2} \\
& +v_{1}|\varphi|^{2} \sum_{a=1}^{3} \sum_{i=1}^{3}\left|\phi_{a}^{i}\right|^{2}-v_{2}|\varphi|^{2} \sum_{a=1}^{3}\left|\boldsymbol{\alpha}_{i} \cdot \phi_{a}^{i *}\right|^{2} .
\end{aligned}
$$

The object $\boldsymbol{\alpha}$ is a global vector and is contracted against the multiplicity index $i$ of the scalar fields $\phi^{i}$. A global symmetry of the model allows one to fix the orientation of $\alpha$ and henceforth we shall work with $\boldsymbol{\alpha}=(1,0,0)^{T}$. Following [11], we fix the symmetries of the theory and subsequently parametrise the scalar fields thus:

$\varphi_{r}=\left(\begin{array}{c}\zeta_{w} \\ 0\end{array}\right)$,

$\phi_{a}^{1}=\left(\begin{array}{c}X \cos \delta_{1} \\ 0 \\ 0\end{array}\right)$,

$\phi_{a}^{2}=\left(\begin{array}{c}X \sin \delta_{1} \sin \gamma e^{i \chi_{3}} \\ Y \cos \delta_{2} \\ 0\end{array}\right)$,

$\phi_{a}^{3}=\left(\begin{array}{c}X \sin \delta_{1} \cos \gamma e^{i \chi_{2}} \\ Y \sin \delta_{2} e^{i \chi_{1}} \\ Z\end{array}\right)$,

where the above variables are real and obey the following relationship

$X^{2}+Y^{2}+Z^{2}=\zeta_{s}^{2}$.

The quantities $\zeta_{w}$ and $\zeta_{s}$ are identified with the absolute lengths of the sets of scalar fields:

$\zeta_{w}=\sqrt{|\varphi|^{2}}$

$\zeta_{s}=\sqrt{\left|\phi^{1}\right|^{2}+\left|\phi^{2}\right|^{2}+\left|\phi^{3}\right|^{2}}$.

Expanding the potential $V$ in terms of (8) we obtain

$$
\begin{aligned}
V= & -\mu_{w} \zeta_{w}^{2}+\lambda_{w} \zeta_{w}^{4} \\
& -\mu_{s}\left(X^{2}+Y^{2}+Z^{2}\right)+\lambda_{s}\left(X^{2}+Y^{2}+Z^{2}\right)^{2}
\end{aligned}
$$




$$
\begin{aligned}
& +\kappa_{s}\left[X^{4}+Y^{4}+Z^{4}\right. \\
& +2 X^{2} Y^{2} \sin ^{2} \delta_{1} \cos ^{2} \delta_{2} \sin ^{2} \gamma \\
& +2 X^{2} Y^{2} \sin ^{2} \delta_{1} \sin ^{2} \delta_{2} \cos ^{2} \gamma \\
& +X^{2} Y^{2} \sin ^{2} \delta_{1} \sin 2 \gamma \sin 2 \delta_{2} \cos \left(\chi_{2}-\chi_{1}-\chi_{3}\right) \\
& \left.+2 X^{2} Z^{2} \sin ^{2} \delta_{1} \cos ^{2} \gamma+2 Y^{2} Z^{2} \sin ^{2} \delta_{2}\right] \\
& +v_{1} \zeta_{w}^{2}\left(X^{2}+Y^{2}+Z^{2}\right)-v_{2} \zeta_{w}^{2} X^{2} \cos ^{2} \delta_{1} .
\end{aligned}
$$

For convenience the following quantities are defined

$$
\begin{aligned}
& \Delta_{1}=X^{2}-Y^{2}, \\
& \Delta_{2}=Y^{2}-Z^{2}, \\
& R=\frac{\nu_{2} \zeta_{w}^{2}}{2 \kappa_{s} \zeta_{s}^{2}} .
\end{aligned}
$$

We consider the following critical point of the potential $V$, which we express in terms of $\zeta_{s}, \Delta_{1}$ and $\Delta_{2}$ :

$\chi_{1}=\chi_{2}=\chi_{3}=\delta_{1}=\delta_{2}=\Delta_{2}=0$,

$\Delta_{1}=R$,

$\zeta_{w}^{2}=\frac{3 \mu_{w}+(1+2 R) \nu_{2} \zeta_{s}^{2}-3 v_{1} \zeta_{s}^{2}}{6 \lambda_{w}}$,

$\zeta_{s}^{2}=\frac{3 \mu_{s}-3 v_{1} \zeta_{w}^{2}+v_{2} \zeta_{w}^{2}}{2 \kappa_{s}+6 \lambda_{s}}$.

From (9), (12) and (13) we obtain the relationships

$X=\zeta_{s} \sqrt{\frac{1+2 R}{3}}$,

$Y=Z=\zeta_{s} \sqrt{\frac{1-R}{3}}$.

Since the variables $X, Y$ and $Z$ are real it follows that

$$
-\frac{1}{2} \leq R \leq 1
$$

The Hessian $H$ of the system is constructed as a symmetric matrix in the basis:

$\left\{X, Y, Z, \zeta_{w}, \delta_{1}, \delta_{2}, \gamma, \tau_{1}, \tau_{2}, \tau_{3}\right\}$
The form of the Hessian at the critical point defined by the set of (13) is found to be the following

$$
H=8 \cdot\left(\begin{array}{ccc|c}
N & 0 & 0 & \\
0 & \frac{X^{2}}{4}\left(2 Y^{2} \kappa_{s}+v_{2} \zeta_{w}^{2}\right) & 0 & \mathbf{0} \\
0 & 0 & \frac{Y^{4} \kappa_{s}}{2} & \\
\hline \mathbf{0} & & \mathbf{0}_{4}
\end{array}\right)
$$

where $N$ is a $4 \times 4$ matrix given by

$$
\left(\begin{array}{cccc}
X^{2}\left(\kappa_{s}+\lambda_{s}\right) & X Y \lambda_{s} & X Y \lambda_{s} & \frac{X \zeta_{w}}{2}\left(\nu_{1}-\nu_{2}\right) \\
X Y \lambda_{s} & Y^{2}\left(\kappa_{s}+\lambda_{s}\right) & Y^{2} \lambda_{s} & \frac{Y \zeta_{w} \nu_{1}}{2} \\
X Y \lambda_{s} & Y^{2} \lambda_{s} & Y^{2}\left(\kappa_{s}+\lambda_{s}\right) & \frac{Y \zeta_{w} \nu_{1}}{2} \\
\frac{X \zeta_{w}}{2}\left(\nu_{1}-\nu_{2}\right) & \frac{Y \zeta_{w} \nu_{1}}{2} & \frac{Y \zeta_{w} \nu_{1}}{2} & \zeta_{w}^{2} \lambda_{w}
\end{array}\right)
$$

The Hessian is positive-semidefinite if the submatrix $N$ is positive-semidefinite and the other entries of $H$ are nonnegative:

$0 \leq \frac{X^{2}}{4}\left(2 Y^{2} \kappa_{s}+v_{2} \zeta_{w}^{2}\right)$

$0 \leq \frac{Y^{4} \kappa_{s}}{2}$.

Using (14) it can be seen that these entries are non-negative given the following conditions:

$R \geq-\frac{1}{2}$

$\kappa_{s} \geq 0$.

Note that for certain values of the variables and parameters, the submatrix $N$ is singular; for instance:

$R=1,-\frac{1}{2}, \quad \zeta_{s}=0, \quad$ or $\quad \zeta_{w}=0$.

For brevity we shall consider only the case where $N$ is nonsingular, as the special cases where $N$ is singular can be analysed using the same method and are computationally easier.

Finding the eigenvalues of $N$ analytically is difficult and has not been previously completed. Instead we shall utilise the method detailed in Sect. 2 to analyse the vacuum stability. The submatrix $N$ may be factorised as $N=U^{\dagger} U$, where the form of $U$ is given in

$$
U=\left(\begin{array}{cccc}
\zeta_{s} \sqrt{\frac{(1+2 R)\left(\kappa_{s}+\lambda_{s}\right)}{3}} & \zeta_{s} \sqrt{\frac{(1-R) \lambda_{s}^{2}}{3\left(\kappa_{s}+\lambda_{s}\right)}} & \zeta_{s} \sqrt{\frac{(1-R) \lambda_{s}^{2}}{3\left(\kappa_{s}+\lambda_{s}\right)}} & \frac{\zeta_{w}\left(\nu_{1}-\nu_{2}\right)}{2 \sqrt{\kappa_{s}+\lambda_{s}}} \\
0 & \zeta_{s} \sqrt{\frac{(1-R) \kappa_{s}\left(\kappa_{s}+2 \lambda_{s}\right)}{3\left(\kappa_{s}+\lambda_{s}\right)}} & \zeta_{s} \sqrt{\frac{(1-R) \kappa_{s}^{2} \lambda_{s}^{2}}{3 \kappa_{s}\left(\kappa_{s}+\lambda_{s}\right)\left(\kappa_{s}+2 \lambda_{s}\right)}} & \frac{\zeta_{w}\left(\kappa_{s} \nu_{1}+\lambda_{s} \nu_{2}\right)}{2 \sqrt{\kappa_{s}\left(\kappa_{s}+\lambda_{s}\right)\left(\kappa_{s}+2 \lambda_{s}\right)}} \\
0 & 0 & \zeta_{s} \sqrt{\frac{(1-R) \kappa_{s}\left(\kappa_{s}+3 \lambda_{s}\right)}{3\left(\kappa_{s}+2 \lambda_{s}\right)}} & \frac{\zeta_{w}\left(\kappa_{s} \nu_{1}+\lambda_{s} \nu_{2}\right)}{2 \sqrt{\kappa_{s}\left(\kappa_{s}+2 \lambda_{s}\right)\left(\kappa_{s}+3 \lambda_{s}\right)}} \\
0 & 0 & 0 & \frac{\zeta_{w}}{2} \sqrt{\frac{4 \lambda_{w} \kappa_{s}^{2}+\left(12 \lambda_{s} \lambda_{w}-3 v_{1}^{2}+2 \nu_{2} \nu_{1}-v_{2}^{2}\right) \kappa_{s}-2 \lambda_{s} \nu_{2}^{2}}{\kappa_{s}\left(\kappa_{s}+3 \lambda_{s}\right)}}
\end{array}\right) .
$$


Recall that the quantities $\zeta_{w}$ and $\zeta_{s}$ are identified with lengths and thus are defined to be non-negative. Whence we conclude that the diagonal entries of $U$ are real and positive given the following further conditions:

$\lambda_{s}>-\frac{\kappa_{s}}{3}$,

$\lambda_{w}>\frac{2 v_{2}^{2} \lambda_{s}+\left(3 v_{1}^{2}-2 v_{1} v_{2}+v_{2}^{2}\right) \kappa_{s}}{4 \kappa_{s}\left(\kappa_{s}+3 \lambda_{s}\right)}$.

In which case, by the argument detailed in Sect. 2, the Hessian is positive-semidefinite. Therefore we have shown that the set of semistable vacua is nonempty and furthermore we have derived the provisions, (18) and (19), under which the vacuum configuration is stable.

\section{Concluding remarks}

We have outlined how the Cholesky decomposition may be applied to the Hessian of a system in order to ascertain the stability of a given critical point. In the example considered above it was not practical to find the eigenvalues of the Hessian analytically and the Cholesky decomposition was key to obtaining the stability conditions for the model under consideration.

Although techniques based upon the Cholesky decomposition are established in some mathematical fields, they have not been widely used in theoretical physics for demonstrating vacuum stability or otherwise. One interesting application of the Cholesky decomposition was presented in [12], in the analysis of fermion masses in a wide class of effective low energy models emerging from intersecting D-brane configurations.

Whilst the application of our method is restricted in this note to the analysis of the Chan-Tsou model, it is evident from our calculation that the approach is quite general. This technique is well suited for the study of models of interest in theoretical physics, many of which present a large number of scalar degrees of freedom, and it is expected that the procedure we have presented will find several further applications in particle theory.

Supersymmetric theories are an immediate candidate for applying this technique as they generally involve many complex scalar fields, leading to complicated effective potentials. It is expected that the approach advocated above may be of use in stability analysis and the classification of flat directions in supersymmetric models $[1-5,13,14]$.

A further opportunity for application is the stability analysis of various grand unified theories which feature, for phenomenological reasons, scalar fields in high dimensional representations of the gauge group (see for instance [15-21]). Whilst work has been undertaken to identify stable vacuum configurations in such models [22-27], there remain open problems which might be addressed using the method presented here.
Acknowledgements I would like to thank Tsou Sheung Tsun and Laura Schaposnik for useful discussions. I am also grateful to Fidel Schaposnik and John March-Russell for their comments on a draft of this paper. This work was funded through a doctoral training account from the Engineering and Physical Sciences Research Council, with additional support from Pembroke College, Oxford.

Open Access This article is distributed under the terms of the Creative Commons Attribution Noncommercial License which permits any noncommercial use, distribution, and reproduction in any medium, provided the original author(s) and source are credited.

\section{References}

1. G.F. Giudice, R. Rattazzi, Phys. Rep. 322, 419 (1999). arXiv: hep-ph/9801271

2. K.A. Intriligator, N. Seiberg, D. Shih, J. High Energy Phys. 0604, 021 (2006). arXiv:hep-th/0602239

3. K.A. Intriligator, N. Seiberg, D. Shih, J. High Energy Phys. 0707, 017 (2007). arXiv:hep-th/0703281

4. D. Shih, J. High Energy Phys. 0802, 091 (2008). arXiv:hep-th/0703196

5. D. Marques, F.A. Schaposnik, J. High Energy Phys. 0811, 077 (2008). arXiv:0809.4618 [hep-th]

6. J. Callahan, Advanced Calculus (Springer, Berlin, 2010), p. 228

7. J.H. Kwak, S.P. Hong, Linear Algebra, 2nd edn. (Birkhauser, Basel, 2004), p. 332

8. J. Gentle, Numerical Linear Algebra for Applications in Statistics (Springer, Berlin, 1998), p. 93

9. S. Wolfram, The Mathematica Book, 5th edn. (Wolfram Media, Champaign, 2004), p. 1103

10. A. Heck, Introduction to Maple, 3rd edn. (Springer, Berlin, 2003), p. 648

11. H.-M. Chan, S.T. Tsou, Eur. Phys. J. C 52, 635 (2007). arXiv: hep-ph/0611364

12. G.K. Leontaris, N.D. Vlachos, J. High Energy Phys. 1001, 016 (2010). arXiv:0909.4701 [hep-th]

13. M. Maniatis, A. von Manteuffel, O. Nachtmann, F. Nagel, Eur. Phys. J. C 48, 805 (2006). arXiv:hep-ph/0605184

14. M.A. Luty, J. March-Russell, H. Murayama, Phys. Rev. D 52, 1190 (1995). arXiv:hep-ph/9501233

15. R. Barbieri, D.V. Nanopoulos, D. Wyler, Phys. Lett. B 103, 433 (1981)

16. A. Masiero, D.V. Nanopoulos, K. Tamvakis, T. Yanagida, Phys. Lett. B 115, 380 (1982)

17. B. Grinstein, Nucl. Phys. B 206, 387 (1982)

18. H. Georgi, Phys. Lett. B 108, 283 (1982)

19. D. Chang, R.N. Mohapatra, M.K. Parida, J. Gipson, R.E. Marshak, Phys. Rev. D 31, 1718 (1985)

20. J. Bagger, S. Dimopoulos, E. Masso, M. Hall Reno, Phys. Rev. Lett. 54, 2199 (1985)

21. J. Bagger, S. Dimopoulos, E. Masso, M. Hall Reno, Nucl. Phys. B 258, 565 (1985)

22. J. Basecq, S. Meljanac, D. Pottinger, Nucl. Phys. B 292, 222 (1987)

23. J. Basecq, S. Meljanac, L. O'Raifeartaigh, Phys. Rev. D 39, 3110 (1989)

24. X.G. He, S. Meljanac, Phys. Rev. D 40, 2098 (1989)

25. O. Kaymakcalan, L. Michel, K.C. Wali, W.D. McGlinn, L. O'Raifeartaigh, Nucl. Phys. B 267, 203 (1986)

26. R. Thornburg, W.D. McGlinn, Phys. Rev. D 33, 2991 (1986)

27. L. O'Raifeartaigh, Group Structure of Gauge Theories (Cambridge University Press, Cambridge, 1986) 